\title{
Analysis of the prevalence of dyslipidemia in individuals with HIV and its association with antiretroviral therapy
}

\author{
Talita Gabriela de Limas ${ }^{[1]}$, Gustavo de Araujo Pinto ${ }^{[2]}$, Liz Miriane Marcato[1] \\ and Daniel Reszel Coelho ${ }^{[1]}$
}

[1]. Faculdade de Medicina, Universidade do Sul de Santa Catarina, Palhoça, SC. [2]. Departamento de Doenças Infecciosas, Hospital Regional Doutor Homero de Miranda Gomes, São José, SC.

\begin{abstract}
Introduction: Antiretroviral therapy (ART) has been used to treat large numbers of patients living with human immunodeficiency virus (HIV) infection. Lipid disorders are often observed in these patients, and include elevations in total cholesterol (TC) and triglycerides (TG). Methods: A cross-sectional study was performed using 333 patient records from the Regional Hospital of São José Doutor Homero de Miranda Gomes (HRSJHMG). The study population consisted of patients with HIV who were under medical follow up, either on or off drug treatment. The data were entered into Excel and exported to SPSS 16.0 for analysis using chi-square testing. We used prevalence ratios as the measure of association. Results: Lipid abnormalities were observed in $78.9 \%$ of individuals who received ART. Of the 308 subjects on ART, $59.1 \%, 41.9 \%$, and $33.1 \%$ had TG, TC and low-density lipoprotein (LDL) abnormalities, respectively. The prevalence of LDL changes was 2.57 -fold higher in individuals who had been using ART for more than 12 months, compared to those using ART for 6 to 12 months. Conclusions: HIV patients showed a significant increase in the association between TC and TG levels and the use of ART. In particular, changes in TC, LDL and TG were greater in individuals who had received ART for over more than 12 months.
\end{abstract}

Keywords: Antiretroviral therapy. HIV. Dyslipidemia. AIDS.

\section{INTRODUCTION}

Antiretroviral therapy (ART) has been used to treat large numbers of patients living with human immunodeficiency virus (HIV) infection worldwide. This therapy is effective at decreasing the morbidity and mortality caused by the virus and improves the quality of life of treated individuals ${ }^{1-4}$. The development of new drugs has led to restoration of the immune system and suppression of viral replication in HIV patients, which has brought new perspectives to the treatment of a disease that had previously displayed a rapid and fatal course ${ }^{5-7}$.

However, ART-treated patients show abnormalities in body fat distribution, insulin resistance and an increased risk of cardiovascular disease, such as stroke and coronary artery disease $^{6,7}$. In addition, lipid disorders are often observed in people living with HIV, and include elevations in total cholesterol (TC) and triglycerides (TG) and reduced levels of high-density lipoprotein (HDL) ${ }^{8}$.

\footnotetext{
Address to: Dr- ${ }^{\mathrm{a}}$ Talita Gabriela de Limas. FM/UNISUL. Rua Hildebrando José da Silva 410, 88304-200 Itajaí, SC, Brasil.

Phone: 5547 9941-5497

e-mail: talitalimas.med@hotmail.com

Received 18 June 2014

Accepted 20 October 2014
}

Studies have reported a high prevalence of metabolic syndrome (MS) in HIV-infected patients receiving ART ${ }^{3,9,10}$. Other studies have shown an increased prevalence of MS among HIV-positive patients, although this rate was not higher than that among HIV-negative individuals ${ }^{11,12}$. Thus, some controversy exists with respect to metabolic changes in HIVpositive patients. Studies have likely shown conflicting results because they are limited by factors such as study design, patient selection, statistical analysis, relatively low event rates, and incomplete ascertainment of cases $^{5,13}$.

If it were known whether these metabolic disorders occur due to ART or as a direct effect of the virus in the body, and if we could determine the specific factors associated with changes in the lipid profile, it would then be possible to select adequate care and treatment for HIV-positive patients, either with the use of drugs or through lifestyle changes. Furthermore, it is important to compare potential increases in the lipid profile of HIV patients to other populations previously studied in other cities and regions. Through the analysis of patient history, lipid profile and duration of treatment, this study sought to compare patients on or off ART and analytically address the factors associated with lipid changes in the HIV-positive population.

\section{METHODS}

This cross-sectional observational study was conducted at the Department of Infectious Diseases, Hospital Regional de São José Doutor Homero de Miranda Gomes (HRSJHMG), 
which is located in São José, State of Santa Catarina. The data were obtained from a total of 649 medical records of individuals with HIV treated at HRSJHMG. The study population consisted of 333 patients who were under medical follow up and were either on or off drug treatment. All patients above the age of 18 years were included in the study from the moment they started clinical follow up. The following medical records were excluded: those for diabetic patients; patients who started ART less than six months earlier; those who underwent treatment with lipid-lowering agents; and records that were illegible or lacked data needed for the study.

The selected charts were divided into three groups: patients on ART for more than 6 months and less than 12 months; patients on ART for more than 12 months and patients who did not use ART.

For records with two or more lipid profile test measurements, the mean levels of TC, TG, HDL and low-density lipoprotein (LDL) were calculated. For patients who received ART, we calculated the average lipid profile results obtained after the beginning of treatment. The calculation for LDL was based on the Friedewald formula, $\mathrm{LDL}=\mathrm{TC}-\mathrm{HDL}-(\mathrm{TG} / 5)$, except in patients with TG levels above $400 \mathrm{mg} / \mathrm{dL}$.

The classification of lipid profile results (TC, TG, HDL and LDL) as normal or abnormal was based on the guidelines of the Adult Treatment Panel III ${ }^{14}$.

The data were collected using an instrument specifically developed for this study, which addressed issues related to ART, laboratory data related to lipid profile and demographic variables.

All data were entered in Excel and exported to Statistical Package for the Social Sciences (SPSS) 16.0. In the bivariate analysis, the chi-square test $\left(\chi^{2}\right)$ was applied, with a significance level of 5\%. Prevalence ratios (PR) were used as the measure of association, and the $95 \%$ confidence interval $(95 \% \mathrm{CI})$ was estimated.

\section{Ethical considerations}

This work was based on the ethical principles of Resolution 466/12 of the National Health Council and was approved by the Research Ethics Committee from the Universidade do Sul de Santa Catarina, under registration 12.098.4.01.III.

\section{RESULTS}

The results were obtained from a total of 649 medical records of individuals with HIV treated at HRSJHMG. Of these, 333 records were included in the study.

The demographic characteristics of the study population are shown in Table 1. It was found that $51.7 \%$ of the HIV patients were male and the age of the individuals included in the study ranged from 18 to 74 years, with an average of 43.3 years. The age ranges with the highest rates of HIV positivity were 31-40 years and $41-50$ years (33.9\% and $32.4 \%$, respectively). The prevalence of lipid abnormalities in HIV patients is shown in Table 2. In the study population, $77.2 \%$ of subjects showed
TABLE 1 - Demographic characteristics of the population.

\begin{tabular}{lcc}
\hline Variable & Number & Percentage \\
\hline Sex & 172 & 51.7 \\
male & 161 & 48.3 \\
female & & \\
\hline Age (years) & 33 & 9.9 \\
18 to 30 & 113 & 33.9 \\
31 to 40 & 108 & 32.4 \\
41 to 50 & 58 & 17.4 \\
51 to 60 & 21 & 6.4 \\
$\geq 61$ & 333 & 100.0 \\
\hline Total & & \\
\hline
\end{tabular}

TABLE 2 - Prevalence of lipid abnormalities in HIV patients.

\begin{tabular}{lcc}
\hline Variables & Number & Percentage \\
\hline Lipid abnormality & 76 & 22.8 \\
no & 257 & 77.2 \\
yes & & \\
\hline Number of lipid abnormalities* & 76 & 22.8 \\
none & 63 & 19.0 \\
one & 115 & 34.5 \\
two & 54 & 16.2 \\
three & 25 & 7.5 \\
four & 333 & 100.0 \\
\hline Total &
\end{tabular}

HIV: human immunodeficiency virus. *total cholesterol; triglycerides; high-density lipoprotein; low- density lipoprotein.

lipid abnormalities, and of these, $34.5 \%$ showed alterations in two lipid levels.

The association between the use/duration of ART and lipid changes, including the levels of TG, TC and LDL are shown in Table 3. Of the 333 charts, $92.5 \%$ of subjects received antiretroviral therapy and $77.8 \%$ had been on treatment for over 12 months.

Lipid abnormalities were observed in $78.9 \%$ of individuals who used ART and $82.2 \%$ of those patients had been on treatment for over 12 months. The prevalence of a lipid change was 1.26 times higher in individuals who used ART compared to those off therapy.

Of the 308 subjects on ART, 59.1\% showed TG abnormalities. In the analysis of time on ART, it was noted that the prevalence of abnormal TG was 2.67 times higher in individuals who used ART for more than 12 months, compared to those who did not use ART. 
TABLE 3 - Association between the use/duration of ART and lipid changes, including the levels of TG, TC and LDL.

\begin{tabular}{|c|c|c|c|c|c|c|c|c|}
\hline \multirow[b]{3}{*}{ Variables } & \multicolumn{6}{|c|}{ Lipid abnormality } & \multirow[b]{3}{*}{ p-value } & \multirow[b]{3}{*}{ PR (95\% CI) } \\
\hline & \multicolumn{2}{|c|}{ no change } & \multicolumn{2}{|c|}{ change } & \multicolumn{2}{|c|}{ Total } & & \\
\hline & $\mathrm{n}$ & $\%$ & $\mathrm{n}$ & $\%$ & $\mathrm{n}$ & $\%$ & & \\
\hline \multicolumn{9}{|l|}{ ART use } \\
\hline yes & 65 & 21.1 & 243 & 78.9 & 308 & 92.5 & 0.009 & $1.26(1.19-1.32)$ \\
\hline no use & 11 & 44.0 & 14 & 56.0 & 25 & 7.5 & \multirow[t]{3}{*}{$<0.001$} & 1 \\
\hline 6 to 12 & 19 & 38.8 & 30 & 61.2 & 49 & 14.7 & & $1.09(0.86-1.27)$ \\
\hline over 12 & 46 & 17.8 & 213 & 82.2 & 259 & 77.8 & & $1.46(1.08-1.98)$ \\
\hline \multicolumn{9}{|l|}{ ART use } \\
\hline yes & 126 & 40.9 & 182 & 59.1 & 308 & 92.5 & \multirow[t]{2}{*}{$<0.001$} & $2.46(1.09-5.55)$ \\
\hline no & 19 & 76.0 & 6 & 24.0 & 25 & 7.5 & & 1 \\
\hline \multicolumn{9}{|c|}{ ART duration (months) } \\
\hline no use & 19 & 76.0 & 6 & 24.0 & 25 & 7.5 & \multirow[t]{3}{*}{$<0.001$} & 1 \\
\hline 6 to 12 & 33 & 67.3 & 16 & 32.7 & 49 & 14.7 & & $1.36(0.53-3.47)$ \\
\hline over 12 & 93 & 35.9 & 166 & 64.1 & 259 & 77.8 & & $2.67(1.18-6.03)$ \\
\hline no & 20 & 80.0 & 5 & 20.0 & 25 & 7.5 & 0.032 & 1 \\
\hline ART durati & & & & & & & & \\
\hline no use & 20 & 80.0 & 5 & 20.0 & 25 & 7.5 & $<0.001$ & 1 \\
\hline 6 to 12 & 41 & 83.7 & 8 & 16.3 & 49 & 14.7 & & $0.81(0.22-2.69)$ \\
\hline over 12 & 138 & 59.8 & 121 & 40.2 & 259 & 77.8 & & $2.33(1.27-9.68)$ \\
\hline & & & & & & & & \\
\hline & & & & & & & & \\
\hline Variables & $\mathrm{n}$ & $\%$ & $\mathrm{n}$ & $\%$ & $\mathrm{n}$ & $\%$ & p-value & PR $(95 \% \mathrm{CI})$ \\
\hline ART use & & & & & & & & \\
\hline yes & 206 & 66.9 & 102 & 33.1 & 308 & 92.5 & 0.349 & $1.38(0.60-3.14)$ \\
\hline no & 19 & 76.0 & 6 & 24.0 & 25 & 7.5 & & 1 \\
\hline ART durati & & & & & & & & \\
\hline no use & 19 & 76.0 & 6 & 24.0 & 25 & 7.5 & 0.006 & $1.68(0.56-5.00)$ \\
\hline 6 to 12 & 42 & 85.7 & 7 & 14.3 & 49 & 14.7 & & 1 \\
\hline over 12 & 164 & 63.3 & 95 & 36.7 & 259 & 77.8 & & $2.57(1.19-5.83)$ \\
\hline
\end{tabular}

ART: antiretroviral therapy; TG: triglycerides; TC: total colesterol; LDL: low-density lipoprotein; PR: prevalence ratio; $95 \%$ CI: $95 \%$ confidence intervals. 
For the association between the duration of ART use and elevated HDL levels, it was found that $38.6 \%$ of subjects on ART had abnormal HDL levels. Regarding the duration of ART, we observed that in individuals on treatment for over 12 months, there was a lower rate of change in the HDL level (39.4\%) when compared to individuals who did not use HIV drugs $(44 \%)$. However, there was no statistical significance between the analyzed variables.

In total, $41.9 \%$ of subjects on ART demonstrated alterations in TC ( $p=0.032)$. The prevalence of TC changes was 2.33 fold higher in individuals who had been using ART for more than 12 months, compared to those off ART.

Regarding LDL levels, individuals who used ART showed a $33.1 \%$ change. The prevalence of an altered LDL level was 2.57-fold higher in individuals who had been using ART for more than 12 months, compared to those using ART between 6 and 12 months.

\section{DISCUSSION}

One strength of this study was that it was conducted in a referral center for HIV care. On the other hand, there were limitations that were beyond our control. For instance, the lipid profile of patients who were not on ART was not requested in most cases, generating a reduced sample that prevented the formation of a more significant group, that would have allowed for a more discriminative and comparative statistical analysis.

In terms of the demographic characteristics of the population, there was a slightly higher prevalence of males with HIV. The mean age of the subjects included in the study was 43.3 years, similar to values reported in the literature ${ }^{1,3,7,8,15-18}$.

The results of this study reinforce the notion that ART is associated with lipid profile changes in patients with HIV $8,15,17-20$. Because ART regimens lead to changes in the lipid profile of HIV patients, there is controversy as to how to determine the best drug regimen in regards to the patient's metabolic profile.

Among the study population, the majority of patients showed lipid changes, and of these, approximately one third of patients showed alterations in two lipid measurements. Among individuals who received ART, 78.9\% showed lipid profile abnormalities. Similarly, Calza et al. ${ }^{21}$ reported that dyslipidemia was detected in up to $70-80 \%$ of HIV-infected individuals on ART, and such changes may be associated with different types of treatment regimens adopted.

The frequency of lipid abnormalities in this study may be explained by the fact that all patients using lipid lowering drugs were excluded from the study, which allowed for a more accurate representation of the data. Other reasons that may result in greater lipid changes include aging, increased body mass index, smoking and the direct effect of HIV infection.

Several studies have provided evidence for the role of ART in the development of metabolic disorders, specifically demonstrating a greater prevalence of hypercholesterolemia and hypertriglyceridemia in patients receiving ART $^{9,15-18,20,21}$.
In the present study, most HIV patients showed statistically significant increases in the association between TC and TG and the use of ART. This association was not found when HDL was examined. Similarly, Araújo et al. ${ }^{15}$ found an association between elevated levels of TG and TC and the use of ART, but no association with HDL.

Although viral load was not part of the analysis in this study, one possible explanation for the finding of higher HDL levels in patients on ART is that they could have a lower viral load, leading to a diminished inflammatory response ${ }^{1}$.

Differences reported in the literature related to the association between ART and changes in the lipid profile, may be attributed to diet, sex, age, physical activity, type and/or duration of ART, as well as differences in the methodology used and the characteristics of each study population.

When examining the association between duration of ART use and the development of lipid abnormalities in the study population, it was observed that the prevalence of abnormalities in TC and TG was greater in patients who had received ART for more than 12 months compared to those not on treatment.

Previous studies have shown that individuals with MS had a longer period of $\mathrm{ART}^{9,22,23}$. On the other hand, Mondy et al. ${ }^{12}$ found no statistical association between use/duration of ART and dyslipidemia. This difference could be explained by patients on ART who also received treatment with lipid-lowering drugs.

This study demonstrated that the prevalence of lipid abnormalities in HIV patients tends to increase according to the duration of ART. Therefore, individuals on ART may have a higher risk of developing cardiovascular disease and atherosclerosis compared to the general population and patients off treatment.

However, the pathophysiological mechanism for such metabolic changes has not been fully established ${ }^{24}$ and prospective studies with longer follow-up periods will provide additional data on the metabolic consequences of ART.

To prevent or lessen the impact of dyslipidemia on the health of individuals with HIV, potential treatment approaches include the switch to of highly active antiretroviral therapy (HAART), prescription of lipid-lowering drugs and/or modification of lifestyle, such as increased physical activity, weight reduction, and decreased smoking and alcohol consumption.

Most of the HIV patients included in this study showed lipid alterations, with approximately one third of these patients showing alterations in two lipid parameters. The HIV patients in our study also demonstrated a statistically significant increase in the association between TC and TG levels and the use of ART. Furthermore, regarding the association between the duration of ART and the development of lipid abnormalities, it was observed that the prevalence of abnormalities in TC, LDL and TG was greater in patients who had received ART for more than 12 months when compared to those not on treatment.

\section{CONFLICT OF INTEREST}

The authors declare that there is no conflict of interest. 


\section{REFERENCES}

1. Hammer SM, Squires KE, Hughes MD, Grimes JM, Demeter LM, Currier JS, et al. A controlled trial of two nucleoside analogues plus indinavir in persons with human immunodeficiency virus infection and CD4 cell counts of 200 per cubic millimeter or less. AIDS Clinical Trials Group 320 Study Team. N Engl J Med 1997; 337:725-733.

2. Gortmaker SL, Hughes M, Cervia J, Brady M, Johnson GM, Seage GR $3^{\text {rd }}$, et al. Effect of combination therapy including protease inhibitors on mortality among children and adolescents infected with HIV-1. N Engl J Med 2001; 345:1522-1528.

3. Jericó C, Knobel H, Montero M, Ordoñez-Llanos J, Guelar A, Gimeno JL, et al. Metabolic syndrome among HIV-infected patients: prevalence, characteristics, and related factors. Diab Care 2005; 28:144-149.

4. Amorosa V, Synnestvedt M, Gross R, Friedman H, MacGregor RR, Gudonis D, et al. A tale of 2 epidemics: the intersection between obesity and HIV infection in Philadelphia. J Acquir Immune Defic Syndr 2005; 39:557-561.

5. Samaras K. Metabolic consequences and therapeutic options in highly active antiretroviral therapy in human immunodeficiency virus-1 infection. J Antimicrob Chemother 2008; 61:238-245.

6. Grinspoon S, Carr A. Cardiovascular risk and body-fat abnormalities in HIV-infected adults. N Engl J Med 2005; 352:48-62.

7. Mulligan K, Grunfeld C, Tai VW, Algren H, Pang M, Chernoff DN, et al. Hyperlipidemia and insulin resistance are induced by protease inhibitors independent of changes in body composition in patients with HIV infection. J Acquir Immune Defic Syndr 2000; 23:35-43.

8. Estrada V, Geijo P, Fuentes-Ferrer M, Alcalde MLG, Rodrigo M, Galindo MJ, et al. Dyslipidaemia in HIV-infected women on antiretroviral therapy. Analysis of 922 patients from the Spanish VACH cohort. BMC Women's Health 2011; 11:36.

9. Samaras K, Wand H, Law M, Emery S, Cooper D, Carr A. Prevalence of metabolic syndrome in HIV-infected patients receiving highly active antiretroviral therapy using International Diabetes Foundation and Adult Treatment Panel III Criteria. Diab Care 2007; 30:113-119.

10. Palella F, Wang Z, Chu H, Riddler S, Visscher B, Dobs A, et al. Correlates and prevalence of the metabolic syndrome over time in the Multicenter AIDS Cohort Study (MACS). Program and Abstracts. $3^{\text {rd }}$ IAS Conference on HIV Pathogenesis and Treatment (Rio de Janeiro, Brazil); 2005.

11. May M, Sterne JAC, Shipley M, Brunner E, d'Agostino R, Whincup $\mathrm{P}$, et al. A coronary heart disease risk model for predicting the effect of potent antiretroviral therapy in HIV-1 infected men. Int J Epide 2007; 36:1309-1318.
12. Mondy K, Overton ET, Grubb J, Tong S, Seyfried W, Powderly W, et al. Metabolic syndrome in HIV-infected patients from an urban, midwestern US outpatient population. Clin Infect Dis 2007; 44:726-734.

13. Silva EFR, Bassichetto KC, Lewi DS. Perfil lipídico, fatores de risco cardiovascular e Síndrome Metabólica em um Grupo de Pacientes com AIDS. Arq Bras Cardiol 2009; 93:113-118.

14. Third report of the National Cholesterol Education Program (NCEP) Expert Panel on Detection, Evaluation, and Treatment of High Blood Cholesterol in Adults (Adult Treatment Panel III) final report. Circulation 2002; 106:3143-3121.

15. Araújo PSR, Ximenes RAA, Lopes CFC, Duarte JY, Silva MM, Carneiro EM. Antiretroviral treatment for HIV infection/AIDS and the risk of developing hyperglycemia and hyperlipidemia. Rev Inst Med Trop São Paulo 2007; 49:73-78.

16. Almeida SEM, Borges M, Fiegenbaum M, Nunes CC, Rossetti MLR. Metabolic changes associated with antiretroviral therapy in HIVpositive patients. Rev Saude Publica 2009; 43:283-290.

17. Silva EFR, Bassichetto KC, Lewi DS. Perfil lipídico, fatores de risco cardiovascular e Síndrome Metabólica em um Grupo de Pacientes com AIDS. Arq Bras Cardiol 2009; 93:113-118.

18. Farhi L, Lima DB, Cunha CB. Dislipidemia em pacientes HIV/AIDS em uso de anti-retrovirais num hospital universitário, Rio de Janeiro, Brasil. J Bras Patol Med Lab 2008; 44:175-184.

19. Riddler SA, Smit E, Cole SR, Li R, Chmiel JS, Dobs A, et al. Impact of HIV infection and HAART on serum lipids in men. JAMA 2003; 289:2978-2982.

20. Friis-Møller N, Weber R, Reiss P, Thiébaut R, Kirk O, d'Arminio Monforte A, et al. Cardiovascular disease risk factors in HIV patientsassociation with antiretroviral therapy. Results from the DAD study. AIDS 2003; 17:1179-1193.

21. Calza L, Manfredi R, Chiodo F. Dyslipidaemia associated with antiretroviral therapy in HIV-infected patients. Antimicrob Chemother 2004; 53:10-14.

22. Palacios R, Santos J, Gonzalez M, Ruiz J, Marquez M. Incidence and prevalence of the metabolic syndrome in a cohort of naive HIV-infected patients: prospective analysis at 48 weeks of highly active antiretroviral therapy. AIDS 2007; 18:184-187.

23. Wand H, Calmy A, Carey DL, Samaras K, Carr A, Law MG, et al. Metabolic syndrome, cardiovascular disease and type 2 diabetes mellitus after initiation of antiretroviral therapy in HIV-infection. AIDS 2007; 21:2445-2553.

24. Kramer AS, Lazzarotto AR, Sprinz E, Manfroi WC. Alterações metabólicas, terapia antirretroviral e doença cardiovascular em idosos portadores de HIV. Arq Bras Cardiol 2009; 93:561-568. 